\title{
Bronchial Fistula and Pneumomediastinum after EBUS-TBNA Following Mediastinoscopy
}

\author{
Ioannis Bougioukas ${ }^{1}$ Ralf Seipelt ${ }^{1}$ Hanno Huwer $^{1}$ \\ ${ }^{1}$ Department of Cardiothoracic Surgery, SHG-Kliniken Volklingen, \\ Voelklingen, Germany

\begin{abstract}
Address for correspondence loannis Bougioukas, MD, Department of Cardiothoracic Surgery, SHG-Kliniken Volklingen, Richardstr. 5-9, Voelklingen 66333, Germany (e-mail: ybougioukas@yahoo.com).
\end{abstract}

Thorac Cardiovasc Surg Rep 2019;8:e11-e13.

\author{
Abstract \\ Keywords \\ - mediastinum (incl. \\ mediastinoscopy) \\ - thoracic surgery \\ - mediastinal lymph \\ nodes \\ - ultrasound \\ (all applications)
}

Background Endobronchial ultrasound-guided transbronchial needle aspiration (EBUS-TBNA) is a very useful diagnostic tool for the assessment of enlarged mediastinal and hilar lymph nodes. It is a safe procedure with a low risk of complications. Case Description We report a case of bronchial fistula and pneumomediastinum after EBUS-TBNA, which was performed shortly after a mediastinoscopy. Due to the extent of the bronchial lesion, a surgical closure of the bronchial fistula was necessary. The patient recovered completely.

Conclusion The performance of EBUS-TBNA shortly after a mediastinoscopy should not be recommended to avoid possible procedure-related complications.

\section{Introduction}

Endobronchial ultrasound-guided transbronchial needle aspiration (EBUS-TBNA) is a widely used diagnostic tool for the assessment of enlarged mediastinal and hilar lymph nodes and therefore plays a substantial role in the staging of lung cancer. ${ }^{1}$ It is a noninvasive and safe technique with a low risk of complications, most of which are relatively harmless, such as cough, agitation, or bleeding at the puncture site. ${ }^{2}$ However, cases of more serious procedure-related events have been sporadically reported in the literature.,

\section{Case Description}

This is a report of a 56-year-old female patient who was admitted at our institution for the clarification of diffuse interstitial infiltrations of the lower lung lobes and hilar and mediastinal lymphadenopathy. A malignancy, in particular a non-Hodgkin lymphoma, was initially suspected and a mediastinoscopy was primarily performed to gain a sufficient amount of material. A bleeding which occurred during this procedure was handled with local compression with a gauge and minimal use of local electrocautery. The procedure was concluded with sampling of the enlarged lymph nodes at the right inferior paratracheal level without complete resection.
Radiological control on the first postoperative day excluded hematoma formation or pneumomediastinum and the patient was discharged on the next day. The histological examination of the specimens showed a histiocytic lymphadenitis. Due to the discrepancy between clinical evaluation and histological findings, an EBUS-TBNA was performed 2 weeks after the mediastinoscopy. Probes were attained from lymph nodes at stations $4 \mathrm{~L}, 4 \mathrm{R}, 11 \mathrm{~L}, 7$ as well as from the right main bronchus.

One week later the patient was readmitted to the hospital due to fever and fatigue. The mediastinoscopy wound was lightly reddened and a small amount of serous fluid was easily evacuated after applying slight pressure on the wound. A computed tomography scan of the thorax was suspicious of a bronchomediastinal fistula depicting a pneumomediastinum (-Figs. 1 and 2), whereas a bronchoscopy confirmed the fistula showing a melting lesion on the right main bronchus (-Fig. 3).

The patient was then subjected to a right axillary thoracotomy; the pretracheal area until the tracheal bifurcation was partially loose but did not have any sign of an extended infection. Microbiological specimens from the site of the paratracheal tissue obtained during the surgical revision were negative. Specimens taken from the lesion site during the preoperative bronchoscopy were positive for Staphylococcus. received

December 9, 2018 accepted after revision March 13, 2019
DOI https://doi.org/

10.1055/s-0039-1688476. ISSN 2194-7635.
๑) 2019 Georg Thieme Verlag KG
Stuttgart · New York

License terms

(1) $\circledast \circledast$ 


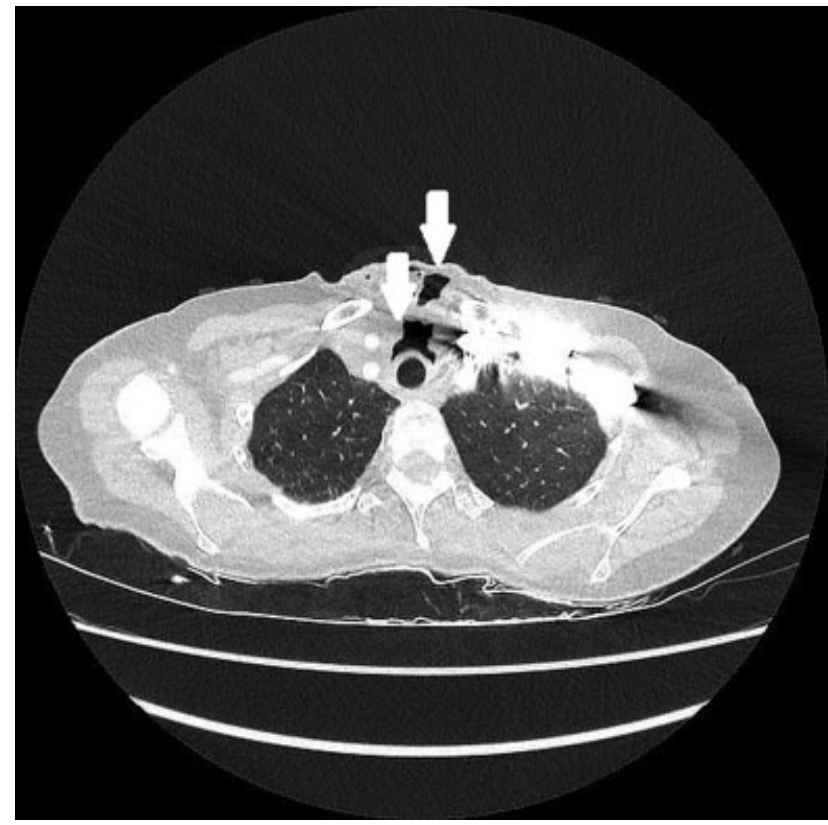

Fig. 1 Computed tomography scan of the thorax following mediastinoscopy and endobronchial ultrasound-guided transbronchial needle aspiration. The white arrows show a tissue dehiscence after spontaneous evacuation of a small amount of fluid from the mediastinoscopy wound.

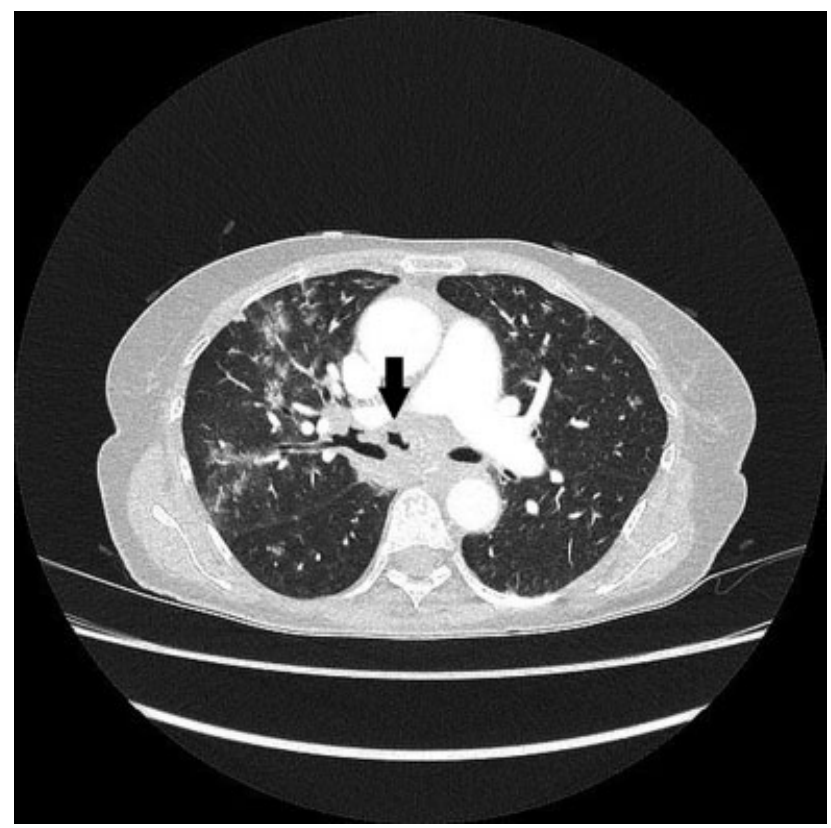

Fig. 2 Computed tomography thoracic scan. The black arrow marks the pneumomediastinum in the area of the fistula on the right upper lobar bronchus.

The fistula with a size of a baby fingertip was located on the right main bronchus. A local debridement was done and the bronchial defect was directly closed with 4-0 PDS sutures and covered with the mobilized azygous vein. An extended biopsy of the enlarged lymph nodes was also performed. The patient was treated with antibiotics postoperatively. Postoperative bronchoscopy revealed a firm closure of the lesion (-Fig. 4). The mediastinoscopy wound healed easily without any further surgical measures.

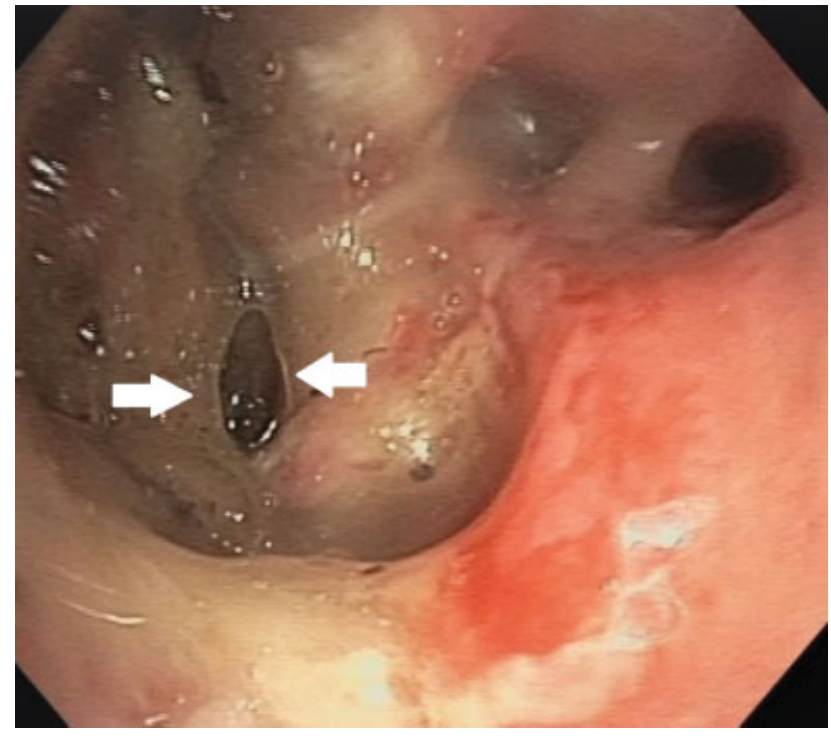

Fig. 3 Bronchoscopical finding depicting the lesion on the right main bronchus (white arrows).

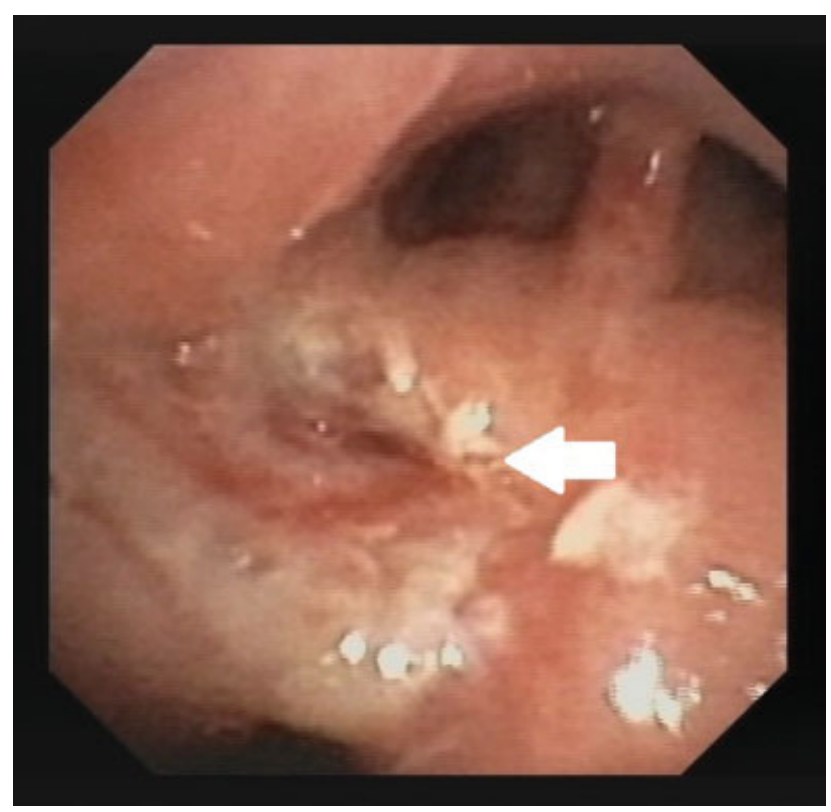

Fig. 4 Bronchoscopical control after lesion repair showing complete closure (white arrow).

The patient recovered completely and was discharged 1 week later. Histology of the abundant specimens excluded again malignancy, showing anthracotic and histiocytic-altered lymphatic tissue, as well as an extended active perilymphadenitis matching to the local bronchomediastinal fistula.

\section{Comment}

In our case, the local tumor board decided for a biopsy of the enlarged mediastinal lymph nodes via mediastinoscopy, as its location was ideal to reach with the conventional mediastinoscope and due to the suspicion of a lymphoma. In cases of lymphoma, abundant tissue sampling is of paramount importance. Farmer et al have already indicated that the reliability of 
diagnosing of lymphoma based on small samples is significantly influenced by the various subtypes. ${ }^{5}$ Though EBUSTBNA offers tissue sampling with minimal trauma, mediastinoscopy has a higher diagnostic accuracy and can be recommended for patients with mediastinal lymphadenopathy or mediastinal mass as first choice diagnostic tool. ${ }^{6}$

A bleeding that occurred during the mediastinoscopy was handled by local compression without excessive use of electrocautery. Furthermore, only sampling of the lymph nodes was performed. The pretracheal space was completely prepared till the tracheal bifurcation, but the area of the right main bronchus was not sampled at the time of the mediastinoscopy. The histological findings excluded malignancy but because of the strong suspicion of a non-Hodgkin lymphoma, a second biopsy via EBUS-TBNA was proposed.

The lesion on the right main bronchus resulted in a local fistula and pneumomediastinum, which had to be managed surgically. Chest X-ray after mediastinoscopy was negative for existence of free air in the mediastinum, whereas at the time of the EBUS-TBNA no lesion was seen on the right bronchial tree. The patient was clinically completely uneventful in the postoperative period after the mediastinoscopy, whereas the symptoms occurred after the TBNA making a clinical correlation with the later formation of the bronchial fistula.

EBUS-TBNA is a safe and accurate diagnostic procedure. Several reports of periprocedural complications can be found in the literature, with an incidence of $\sim 1 \%{ }^{2}$ The vast majority of such complications are not serious and rarely require any invasive measures. ${ }^{2,3,7}$ Pneumomediastinum is a known rare complication after needle trauma of the bronchial wall during EBUS-TBNA. ${ }^{3}$ In general, pneumomediastinum is self-healing because the mediastinal tissue is tight enough to provide resistance against the airflow generated by the intrabronchial pressure. ${ }^{8}$

In our case and here lies a self-criticism, the pretracheal space, which was already surgically prepared during the mediastinoscopy and therefore relatively loose, was completely filled with air which drained off through the collar incision and a fistula from the bronchus through the mediastinum could be established. In any other case, the firmness of the surrounding tissues would probably prevent the formation of a fistula after a similar bronchial lesion.

The bleeding that occurred by the time of the mediastinoscopy was located on the anterior surface of the tracheal bifurcation most probably from a bronchial artery crossing the trachea from the left to the right. Although this bleeding was managed with compression and limited electrocautery, it could have played a role in the later occurrence of the fistula due to a local ischemia of the external bronchial wall. This in combination with a biopsy from the region 2 weeks later could have been the causative mechanism for the emergence of the fistula. Nevertheless, the lesion could be directly closed unproblematically.

In conclusion, although the need of an EBUS-TBNA after a mediastinoscopy is rather unlikely, this should not be performed shortly after the mediastinoscopy, to avoid possible procedure-related local complications, necessitating more invasive treatment.

\section{Conflict of Interest}

None declared.

\section{References}

1 Labarca G, Aravena C, Ortega F, et al. Minimally invasive methods for staging in lung cancer: systematic review and meta-analysis. Pulm Med 2016;2016:1024709

2 Asano F, Aoe M, Ohsaki Y, et al. Complications associated with endobronchial ultrasound-guided transbronchial needle aspiration: a nationwide survey by the Japan Society for Respiratory Endoscopy. Respir Res 2013;14:50

3 Piroddi IMG, Gatto P, Perazzo A, Barlascini C, Nicolini A. Pneumomediastinum following endobronchial ultrasound-guided transbronchial needle aspiration: a case report. Tanaffos 2017; 16(03):245-247

4 Gamrekeli A, Kalweit G, Schäfer H, Huwer H. Infection of a bronchogenic cyst after ultrasonography-guided fine needle aspiration. Ann Thorac Surg 2013;95(06):2154-2155

5 Farmer PL, Bailey DJ, Burns BF, Day A, LeBrun DP. The reliability of lymphoma diagnosis in small tissue samples is heavily influenced by lymphoma subtype. Am J Clin Pathol 2007;128 (03):474-480

6 Zhu F, Ma DC, Xu N, et al. Diagnostic value of video-assisted mediastinoscopy and endobronchial ultrasound-guided transbronchial needle aspiration for mediastinal lymphadenectasis without pulmonary abnormalities. Med Sci Monit 2017; 23:3064-3070

7 Eapen GA, Shah AM, Lei X, et al; American College of Chest Physicians Quality Improvement Registry, Education, and Evaluation (AQuIRE) Participants. Complications, consequences, and practice patterns of endobronchial ultrasound-guided transbronchial needle aspiration: results of the AQuIRE registry. Chest 2013;143(04):1044-1053

8 Kouritas VK, Papagiannopoulos K, Lazaridis G, et al. Pneumomediastinum. J Thorac Dis 2015;7(Suppl 1):S44-S49 